TITLE:

\title{
Low-frequency discrete breathers in long-range systems without on- site potential
}

AUTHOR(S):

Yamaguchi, Yoshiyuki Y.; Doi, Yusuke

\section{CITATION:}

Yamaguchi, Yoshiyuki Y....[et al]. Low-frequency discrete breathers in long-range systems without on-site potential. Physical Review E 2018, 97(6): 062218.

\section{ISSUE DATE:}

2018-06-25

URL:

http://hdl.handle.net/2433/232587

RIGHT:

C2018 American Physical Society 


\title{
Low-frequency discrete breathers in long-range systems without on-site potential
}

\author{
Yoshiyuki Y. Yamaguchi* \\ Department of Applied Mathematics and Physics, Graduate School of Informatics, Kyoto University, Kyoto 606-8501, Japan \\ Yusuke Doi ${ }^{\dagger}$ \\ Department of Adaptive Machine Systems, Graduate School of Engineering, Osaka University, 2-1 Yamadaoka, Suita, Osaka 565-0871, Japan
}

(Received 25 October 2017; published 25 June 2018)

\begin{abstract}
A mechanism of long-range couplings is proposed to realize low-frequency discrete breathers without on-site potentials. The realization of such discrete breathers requires a gap below the band of linear eigenfrequencies. Under the periodic boundary condition of a one-dimensional lattice and the limit of large population, we show theoretically that the long-range couplings universally open the gap below the band irrespective of the coupling functions, while the short-range couplings cannot. The existence of the low-frequency discrete breathers, spatial localization, and stability are numerically analyzed from long range to short range.
\end{abstract}

DOI: 10.1103/PhysRevE.97.062218

\section{INTRODUCTION}

The discrete breather (DB) or the intrinsic localized mode is a solution which is spatially localized as well as temporally periodic, in a spatially discrete system [1-7]. In this article we focus on the following factors to realize the localization: spatial discreteness and nonlinearity of couplings. The discreteness suppresses the band of eigenfrequencies of the linearized system in a bounded range and the nonlinearity provides a frequency outside of the band. Thus, once a localized oscillation is excited, it can be maintained without receiving dispersion by the linear modes. Because of the simple mechanism, DBs are observed in various practical systems of electrical lattices [8,9], mechanical systems $[10,11]$, and granular crystals $[12,13]$ and are expected in crystals $[14,15]$ and spin lattices [16-20].

Discrete breathers have been mainly investigated in shortrange lattice systems having, typically, the nearest-neighbor interaction. However, long-range interacting systems also form lattice structures as vortices of plasmas [21,22] and of fluids [23]. A remarkable example is trapped ions, because the range of interaction can be experimentally varied from the short range to the long range [24-28]. The coupling constants damp as $1 / r^{\alpha}(0 \leqslant \alpha \leqslant 3)$, where $r$ is the distance between the two interacting ions.

The trapped ions have attracted great interest from the viewpoint of the information propagation speed in long-range systems. The long-range nature $(\alpha<D$ in the $D$-dimensional space) breaks [28-30] the Lieb-Robinson bound [31,32], which holds in short-range systems. Discrete breathers, which are spatially localized modes, may concern the information propagation, as we may expect that a standing DB provides no or weak propagation and a traveling DB $[33,34]$ provides the ballistic propagation. Thus, the information propagation

\footnotetext{
*yyama@amp.i.kyoto-u.ac.jp
}

†doi@ams.eng.osaka-u.ac.jp provides the first motivation to investigate DBs in long-range systems.

The second motivation is to extend the realization condition of DBs. In a lattice system, each lattice point has a nonlinear oscillator coupling with other oscillators through the twobody coupling potential. The nonlinearity is classified into hard springs and soft springs, where hard (soft) springs have higher (lower) frequency for larger amplitude. Correspondingly, possible frequencies of DBs are above the band with hard springs, or below the band with soft springs. The latter case, however, is in short-range systems, realized only by adding on-site potentials. We will show that the long-range interaction universally induces a gap below the band, which is called the lower gap in this article, without the on-site potentials.

Several studies on DBs have been performed in lattices with algebraically damping coupling constants [35-37], but consideration of the long-range case is lacking (with $D=1$ and $\alpha>1$ in [35], $\alpha=2$ in [36], and $\alpha=3$ in [37]), as is the existence of the lower gap.

It seems strange that the long-range couplings help to make localized modes. We will explain the basic idea of the mechanism from a simple example of the mean-field couplings in Sec. II. Then we show theoretically that the lower gap exists and survives in the limit of large population under the spatially periodic boundary condition in Sec. III. The band structures are exhibited for both the periodic and fixed boundary conditions in Sec. IV to confirm the appearance of the lower band gap only in the long-range case. The existence of low-frequency standing one-DB solutions, spatial localization, and stability is investigated numerically by varying the value of $\alpha$ from long range to short range in Sec. V. Section VI is devoted to a summary and discussion.

\section{LINEARIZED EQUATION AND EIGENFREQUENCIES}

Let us start by sketching the basic idea of having the lower gap without the on-site potential. We consider a generic classical one-dimensional lattice system described by the 
Hamiltonian

$$
H(q, p)=\sum_{j=0}^{N-1} \frac{p_{j}^{2}}{2}+\frac{1}{2 N_{*}} \sum_{j=0}^{N-1} \sum_{k=0}^{N-1} J_{j k} \phi\left(q_{j}-q_{k}\right) .
$$

This Hamiltonian induces the equations of motion as

$$
\ddot{q}_{j}=-\frac{1}{N_{*}} \sum_{k=0}^{N-1} J_{j k} \phi^{\prime}\left(q_{j}-q_{k}\right) \quad(j=0, \ldots, N-1) .
$$

Let the two-body interaction potential $\phi(q)$ be smooth, even, and satisfy the condition $\phi^{\prime \prime}(0)>0$. The coupling constants $J_{j k}$ are, for instance,

$$
J_{j k}= \begin{cases}1 & \text { for } k=j \pm 1 \\ 0 & \text { otherwise }\end{cases}
$$

for the nearest-neighbor couplings and

$$
J_{j k}=1 \quad(j, k=0, \ldots, N-1)
$$

for the mean-field couplings. The prefactor $N_{*}$ is defined by

$$
N_{*}=\frac{1}{N} \sum_{j=0}^{N-1} \sum_{k=0}^{N-1} J_{j k}
$$

and depends on $N$ in general to ensure the extensivity of the potential term. For instance, the mean-field couplings give $N_{*}=N$. The factor $N_{*}$ seems artificial but, dynamically, it can be eliminated by modifying the time scale as

$$
t \rightarrow t / \sqrt{N_{*}} \text {. }
$$

We keep $N_{*}$ for convenience in discussing the limit $N \rightarrow \infty$.

The evenness of $\phi(q)$ implies $\phi^{\prime}(0)=0$ and hence

$$
q_{j}=0 \quad(j=0, \ldots, N-1)
$$

is a fixed point. Linearizing the equations of motion (2) around this fixed point (7), we obtain

$$
\ddot{\xi}_{j}=-\phi^{\prime \prime}(0) \sum_{k=0}^{N-1} A_{j k} \xi_{k}, \quad A_{j k}=\delta_{j k}-\frac{J_{j k}}{N_{*}},
$$

where $\xi_{j}$ is the infinitesimal displacement from the fixed point and $\delta_{j k}$ is the Kronecker delta. We consider the problem if the eigenvalues of the matrix $A=\left(A_{j k}\right)_{j, k=0, \ldots, N-1}$ have the lower gap.

We note that the eigenvalues of the matrix $C=B+c E_{N}$, where $E_{N}$ is the identity matrix of size $N$, are written as the eigenvalues of $B$ added by $c$. Thus, if identical on-site potentials $U(q)$ satisfying $U^{\prime \prime}(0)>0$ are applied to all the particles, $U^{\prime \prime}(0)$ is uniformly added to the eigenvalues of the matrix $\phi^{\prime \prime}(0) A$. Consequently, the on-site potentials may open the lower gap. We will show in Sec. III that the long-range interaction opens the lower gap without the on-site potentials, while the short-range interaction cannot do that.

The idea to have the lower gap in long-range systems without the on-site potentials is as follows. One typical longrange coupling is the mean-field coupling and the matrix $A$ becomes

$$
A_{\mathrm{MF}}=E_{N}-\frac{1}{N}\left(\begin{array}{ccc}
1 & 1 & \cdots \\
1 & 1 & \cdots \\
\vdots & \vdots & \ddots
\end{array}\right)
$$

The second term of $A_{\mathrm{MF}}$ has rank 1 and all the eigenvalues are zeros except for one -1 . Recalling the previous remark on the eigenvalues, all the eigenvalues of $A_{\mathrm{MF}}$ are 1 except for one 0 corresponding to the total momentum conservation. Therefore, the band of $A_{\mathrm{MF}}$ has a clear gap between 0 and 1 . We extend the existence of this lower gap to the long-range case but not mean-field one.

We remark that the diagonal elements in the second term of $A_{\mathrm{MF}}$ come not from the on-site potentials but from the definition of $J_{k k}=1$ for the mean-field couplings (4). They correspond to the self-interaction and therefore they are not essential dynamically. For instance, the degeneracy of $N-1$ eigenvalues holds even if we change the definition of $J_{k k}$ $(k=0, \ldots, N-1)$ as $J_{k k}=c$ with $c \in \mathbb{R}$ arbitrary, although $N_{*}$ changes.

Hereafter, we suppose that the coupling constants depend on the distance $d(j, k)$ between the sites $j$ and $k$ and they are denoted by $J_{d(j, k)}$. The distance is determined depending on the boundary conditions, which are periodic or fixed. We also assume that the lattice spacing is unity.

\section{LOWER GAP UNDER PERIODIC BOUNDARY CONDITION}

In this section we provide two theorems under the periodic boundary condition to reveal a contrast between the short- and long-range systems. The distance $d(j, k)$ is defined by

$$
d(j, k)=\min \{|j-k|, N-|j-k|\} .
$$

We assume that $N$ is even for simplicity. Then the factor $N_{*}$ is also simplified as

$$
N_{*}=J_{0}+2 \sum_{k=1}^{N / 2-1} J_{k}+J_{N / 2} .
$$

The matrix $A$ is circulant and the eigenvalues are obtained as

$\lambda_{j}(N)=1-\frac{1}{N_{*}}\left[J_{0}+2 \sum_{k=1}^{N / 2-1} J_{k} \cos \frac{2 \pi j k}{N}+(-1)^{j} J_{N / 2}\right]$,

where $j=0,1, \ldots, N-1$ and the relation $\lambda_{N-j}=\lambda_{j}$ holds. The smallest eigenvalue is $\lambda_{0}=0$ because the absolute value of the term in square brackets is less than or equal to $N_{*}$ (recall the definition of $N_{*}$ and the fact that $|\cos x| \leqslant 1$ for any real $x$ ). We assume that the second smallest eigenvalues are $\lambda_{1}=\lambda_{N-1}$ and consider $\lambda_{1}(N)$ in the limit $N \rightarrow \infty$. For the coupling constants $J_{k}$, we assume that there exists the natural number $m(\geqslant 1)$ such that

$$
\begin{array}{ll}
0<J_{k}<\infty & \text { for } k=1, \ldots, m, \\
0 \leqslant J_{k}<\infty & \text { otherwise. }
\end{array}
$$

Theorem 1 (short range). Assume (13). Suppose that there exist $\alpha$ and $J$ such that $\alpha>1, J>0$, and $J_{k} \leqslant J / k^{\alpha}(k=$ $1,2, \ldots)$. Then

$$
\lim _{N \rightarrow \infty} \lambda_{1}(N)=0 .
$$

Theorem 2 (long range). Assume (13). Suppose that there exist $\alpha, J$, and $J^{\prime}$ such that $0 \leqslant \alpha \leqslant 1,0<J^{\prime} \leqslant J$, and 


$$
\begin{aligned}
& J^{\prime} / k^{\alpha} \leqslant J_{k} \leqslant J / k^{\alpha}(k=1,2, \ldots) \text {. Then } \\
& \frac{J^{\prime}}{J} 2^{2-\alpha}(1-\alpha) X_{\alpha} \leqslant \lim _{N \rightarrow \infty} \lambda_{1}(N) \leqslant \frac{J}{J^{\prime}} 2^{2-\alpha}(1-\alpha) X_{\alpha},
\end{aligned}
$$

where

$$
X_{\alpha}=\int_{0}^{1 / 2} x^{-\alpha} \sin ^{2}(\pi x) d x
$$

is a finite positive number.

See Appendix A for proofs. Five remarks are in order. First, the nearest-neighbor couplings and the exponentially damping couplings satisfy the assumptions of Theorem 1 and give the vanishing $\lambda_{1}$. Second, Theorem 2 implies that the equalities in (15) hold if the $J_{k}$ are purely algebraically damping as $J_{k}=J / k^{\alpha}$. Third, however, the lower gap may exist even if the coupling constants $J_{k}$ are not purely algebraically damping. For example, $J_{k}=J / k^{\alpha}+\exp (-k)$ is a target of Theorem 2 . This result helps to examine the existence of DBs experimentally. Fourth, the lower gap obtained for $0 \leqslant \alpha<1$ is enhanced if we eliminate the factor $N_{*}$ in (1) by changing the time scale as (6), because the corresponding frequency $\sqrt{\lambda_{1}}$ becomes $\sqrt{N_{*} \lambda_{1}}$ and $N_{*}$ is $O\left(N^{1-\alpha}\right)$. This scaling can be found in (A5). All the eigenvalues share the scaling and ratios between any pairs of eigenvalues are invariant. However, the enhancement of the absolute value of the lower gap $\sqrt{N_{*} \lambda_{1}}$ is helpful to hit the lower gap experimentally. Moreover, this enhancement is naturally expected since the factor $N_{*}$ is introduced artificially to ensure the extensivity of energy. Fifth, to prove Theorems 1 and 2 we used only the matrix $A$, which is determined by the coupling constants $J_{k}$, and the interaction potential $\phi(q)$ is not included. The results obtained are therefore universal for any coupling functions $\phi(q)$ holding $\phi^{\prime \prime}(0)>0$.

\section{BAND STRUCTURE}

For the periodic boundary condition, we showed theoretically the appearance of the lower gap in long-range systems and the nonappearance in short-range systems. Thus, we may expect that the long-range nature also opens the lower gap under the fixed boundary condition. We now demonstrate the validity of these results and expectation by computing the band structure explicitly. For simplicity, we set

$$
J_{d(j, k)}=\frac{1}{d(j, k)^{\alpha}}, \quad \alpha \geqslant 0
$$

for $j \neq k$.

\section{A. Eigenfrequencies under periodic boundary condition}

We set $J_{0}=1$. The scaled eigenfrequencies $\omega_{j}=\lambda_{j}^{1 / 2}$ are obtained from the formula (12) and reported in Fig. 1(a). As we assumed, $\lambda_{1}$ is the second smallest eigenvalue for any value of $\alpha$. The importance of the long-range nature is confirmed from the $\alpha$ dependence of $\omega_{1}$ in Fig. 1(b) reported with the asymptotic value

$$
\lim _{N \rightarrow \infty} \lambda_{1}(N)=2^{2-\alpha}(1-\alpha) \int_{0}^{1 / 2} x^{-\alpha} \sin ^{2}(\pi x) d x .
$$
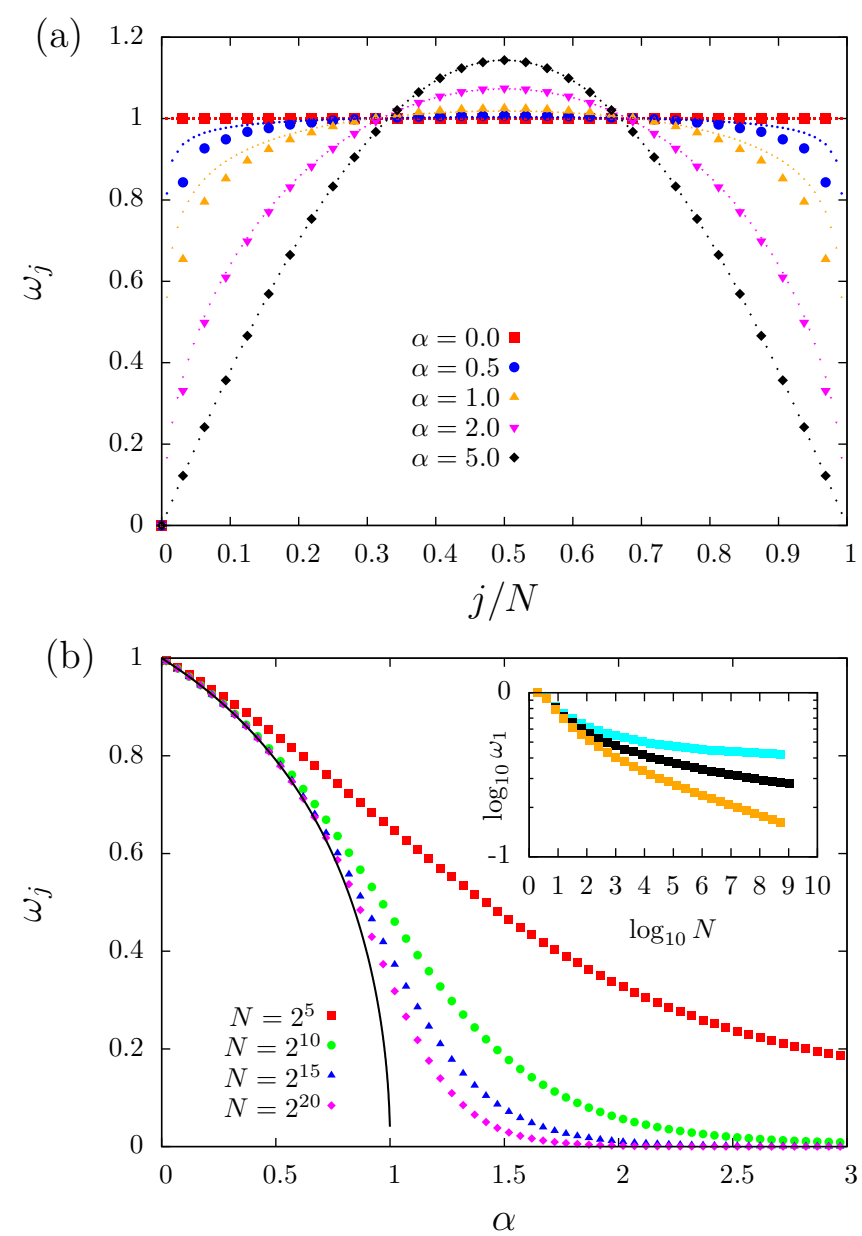

FIG. 1. Scaled eigenfrequencies of the linearized equations of motion, which are square roots of the eigenvalues of the matrix $A$, for the periodic boundary condition. (a) Band structure for several values of $\alpha, N=32$ (large symbols) and $N=128$ (small symbols), and $\omega_{0}=0$ from the total momentum conservation. (b) The $\alpha$ dependence of the smallest positive frequency $\omega_{1}$ for several values of $N$. The black solid curve is obtained theoretically for $N \rightarrow \infty$. The inset shows the $N$ dependence for $\alpha=0.9,1$, and 1.1 from top to bottom.

As the theorems state, the lower gap survives for $\alpha<1$ but it tends to vanish for $\alpha \geqslant 1$ as $N$ increases.

\section{B. Eigenfrequencies under fixed boundary condition}

Under the fixed boundary condition, the distance $d(j, k)$ is simply defined as

$$
d(j, k)=|j-k| .
$$

We set $J_{0}=0$, which differs from the periodic case but demonstrates that this choice is not crucial for the appearance of the lower gap. We fix the -1 th and $N$ th particles, which also have interactions with all the other particles following the rule of coupling constants $J_{d(j, k)}$.

The scaled eigenfrequencies $\omega_{j}$, which are the square roots of the eigenvalues of the matrix $A$, are reported in Fig. 2(a). The fixed particles break the momentum conservation and the lowest eigenvalue of $A$ may not be zero. Thus, to confirm the lower gap, the smallest and second smallest eigenfrequencies 

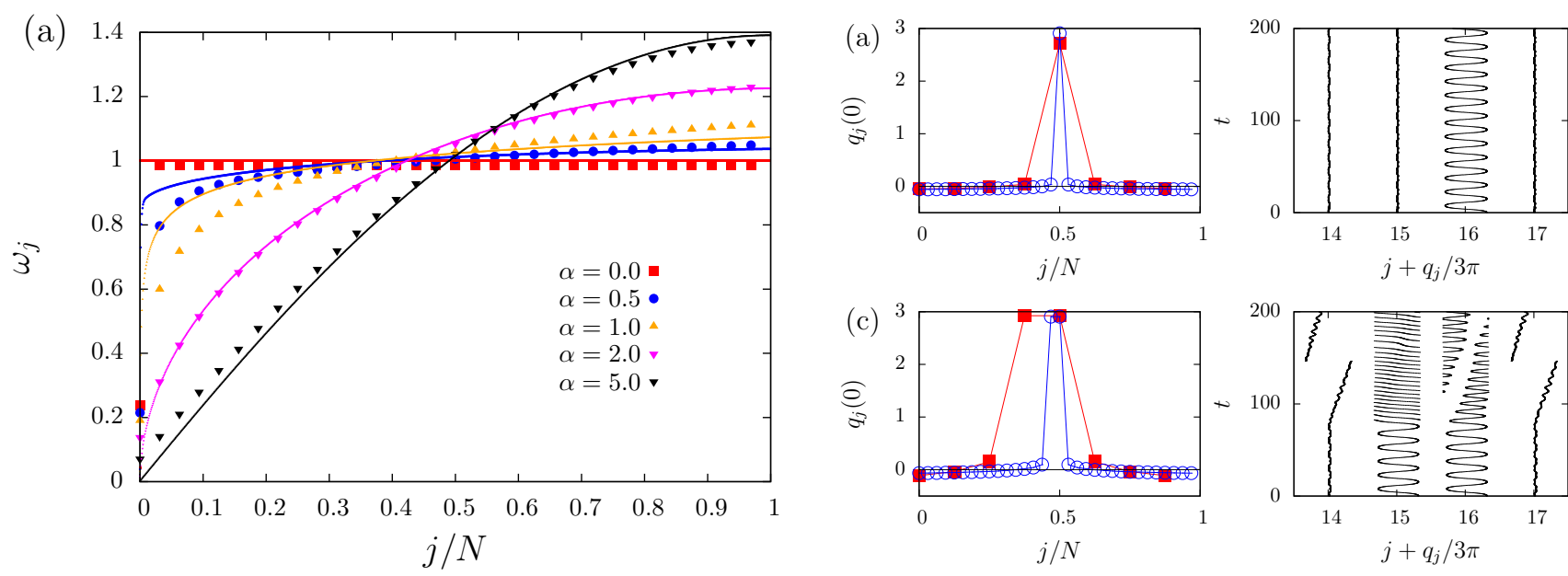

(b)
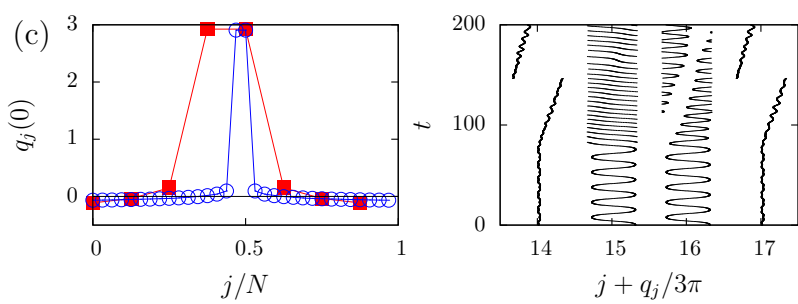

(d)
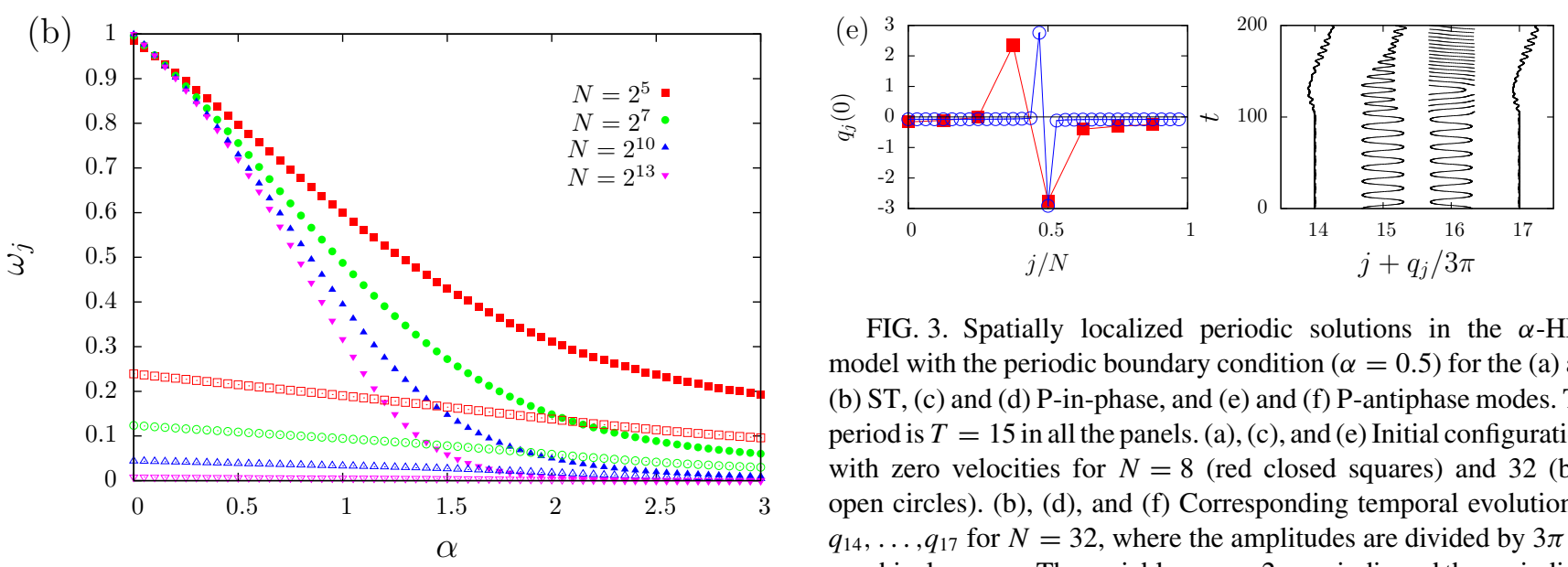

FIG. 3. Spatially localized periodic solutions in the $\alpha$-HMF model with the periodic boundary condition $(\alpha=0.5)$ for the (a) and (b) ST, (c) and (d) P-in-phase, and (e) and (f) P-antiphase modes. The period is $T=15$ in all the panels. (a), (c), and (e) Initial configurations with zero velocities for $N=8$ (red closed squares) and 32 (blue open circles). (b), (d), and (f) Corresponding temporal evolution of $q_{14}, \ldots, q_{17}$ for $N=32$, where the amplitudes are divided by $3 \pi$ for graphical reasons. The variables $q_{k}$ are $2 \pi$ periodic and the periodicity makes artificial discontinuity between $\pi$ and $-\pi$ at some points.

FIG. 2. (a) Same as Fig. 1 but for the xed boundary condition, and for $N=32$ and 1024. (b) Scaled eigenfrequencies $\omega_{0}$ (lower open symbols) and $\omega_{1}$ (upper closed symbols) for several values of $N$ to observe the lower gap.

$\omega_{0}$ and $\omega_{1}$ are reported as functions of $\alpha$ in Fig. 2(b). The lower gap in the limit $N \rightarrow \infty$ can be confirmed only for $\alpha<1$ as the periodic boundary case.

\section{DISCRETE BREATHERS}

We have clarified the appearance of the lower gap in the long-range case. In this section we search for low-frequency DBs by using the lower gap. The stability and $\alpha$ dependence of DBs are also investigated under both the periodic and fixed boundary conditions. As remarked in Sec. III, the lower gap exists for any coupling function $\phi(q)$, but, to hit the lower gap, the interaction should be soft springs: The larger amplitude gives the smaller frequency. To realize the soft springs, we introduce one model for each boundary condition to emphasize the universality of the low-frequency DBs.

The existence of several types of DBs has been proven by considering the anticontinuous limit [38-40]. In this section, however, we focus on the two types of one-DB solutions: the Sievers-Takeno (ST) modes [1] and the Page (P) modes [2], which have odd and even symmetry around the largest amplitude, respectively. In other words, the ST and P modes have the on-site and intersite characterizations, respectively. The $\mathrm{P}$

modes are further classified into the P-in-phase modes and the $\mathrm{P}$-antiphase modes depending on the phase gap between the two particles sharing the largest amplitude. To find the three modes, we performed the shooting method with fixing the target period $T$. See Appendix B for the shooting method.

\section{A. Discrete breathers under periodic boundary condition}

We consider the so-called $\alpha$-Hamiltonian mean-field ( $\alpha$ HMF) model [41], which is described by the Hamiltonian

$$
H_{\mathrm{HMF}}=\sum_{j=0}^{N-1} \frac{p_{j}^{2}}{2}+\frac{1}{2 N_{*}} \sum_{j=0}^{N-1} \sum_{k=0}^{N-1} \frac{1-\cos \left(q_{j}-q_{k}\right)}{d(j, k)^{\alpha}},
$$

where 1 in the potential is added to adjust the potential minimum as 0 . We stress that this system is a classical correspondence of the long-range quantum $X Y$ spin system realized experimentally [28]. The boundary condition is periodic and hence $d(j, k)$ is defined by (10).

We first fix the parameter $\alpha$ as $\alpha=0.5$ and search the periodic solution with the period $T=15$. The corresponding frequency is $\omega \simeq 0.42$, which is in the lower gap as found in Fig. 1(b). We can find spatially localized periodic solutions of the ST, P-in-phase, and P-antiphase modes as exhibited in Fig. 3. 

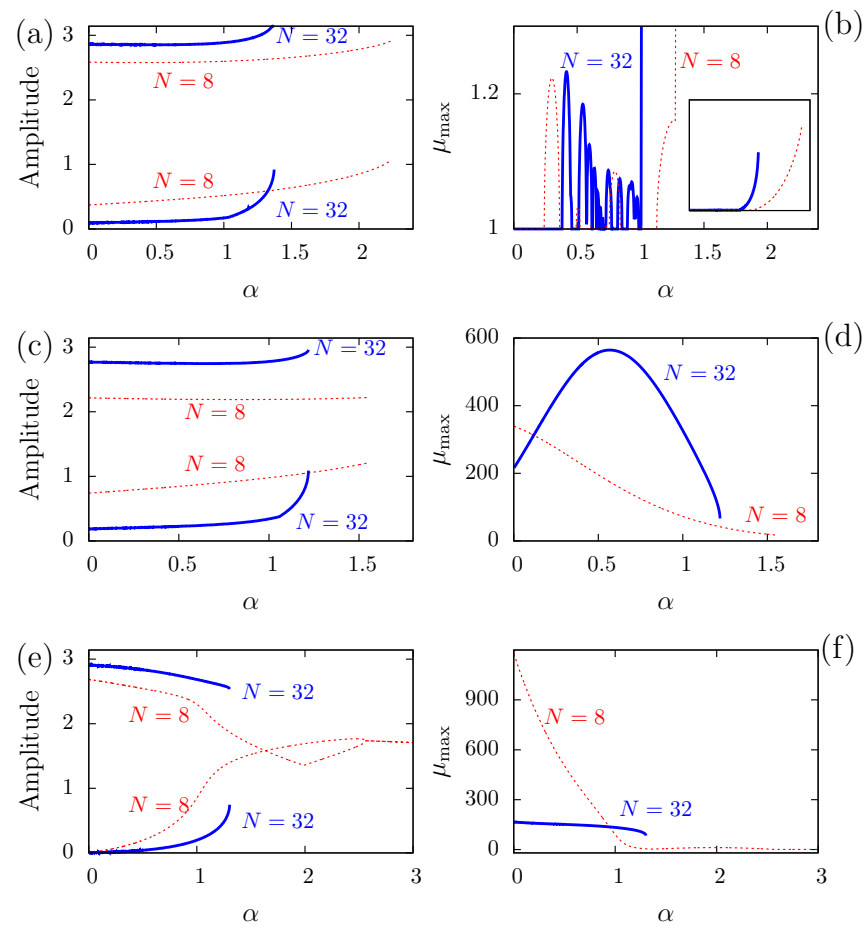

FIG. 4. The $\alpha$ dependence of the (a) and (b) ST, (c) and (d) P-inphase, and (e) and (f) P-antiphase modes for $N=8$ (red thin dotted lines) and $N=32$ (blue thick solid lines). The period is $T=15$ in all the panels. (a), (c), and (e) The first (upper) and second (lower) largest amplitudes. (b), (d), and (f) Maximum absolute value of the characteristic multipliers. The inset in (b) is a distant view, whose horizontal scale is the same as the main panel but the vertical axis shows the interval $[0,300]$.

It is worth commenting that, in the $\alpha$-HMF system, both the $\mathrm{P}$-in-phase and the $\mathrm{P}$-antiphase modes are obtained in the lower gap. This observation is in sharp contrast with the discrete nonlinear Klein-Gordon system, which has the in-phase in the gap below the band, but has the antiphase in the gap above the band [4].

Next we successively vary the value of $\alpha$ from $\alpha=0.5$ and investigate the $\alpha$ dependence of existence, spatial localization, and stability. The step size of $\alpha, \Delta \alpha$, is suitably selected from $10^{3} \Delta \alpha=1,2,3$, and 4 to search periodic solutions as much as possible. The obtained periodic solutions are reported in Fig. 4 and the coexistence of the three modes is observed in $0 \leqslant \alpha \lesssim 1.2$ for $N=32$. We may therefore conclude that coexistence occurs due to the long-range interaction. Some curves stop around $\alpha \simeq 1.2$, beyond which no points are plotted because we could not find periodic solutions based on the above protocol. Of course, we cannot exclude the existence of periodic solutions in larger $\alpha$ but we captured the difficulty of finding spatially localized periodic solutions for large $\alpha$.

The spatial localization is confirmed by computing the first and second largest amplitudes. We note that, from symmetry, the first largest amplitude is shared by one (two) particle(s) in the ST (P) modes and hence the amplitude of the third particle is reported for the $\mathrm{P}$ modes. The localization tends to be enhanced for larger $N$ and for smaller $\alpha$ (longer range of interaction). The stability of the periodic solutions is investigated by computing the maximum absolute value $\mu_{\max }$ of the characteristic multipliers. The periodic solution is marginally stable for $\mu_{\max }=1$, while unstable for $\mu_{\max }>1$. The localized P-in-phase and $\mathrm{P}$-antiphase modes exist for long-range couplings, but they are unstable. In contrast, the instability changes in a complicated manner for the ST mode and drastically increases before the periodic solutions cannot be captured.

\section{B. Discrete breathers under fixed boundary condition}

We introduce a modified Fermi-Pasta-Ulam-Tsingou (FPUT) model $[42,43]$ whose Hamiltonian is expressed as

$$
\begin{aligned}
H_{\mathrm{FPUT}} & =\sum_{j=0}^{N-1} \frac{p_{j}^{2}}{2}+\frac{1}{2 N_{*}} \sum_{j=0}^{N-1} \sum_{k=0}^{N-1} \frac{\phi_{\mathrm{FPUT}}\left(q_{j}-q_{k}\right)}{|j-k|^{\alpha}}, \\
\phi_{\mathrm{FPUT}}(q) & =\frac{q^{2}}{2}+\beta \frac{q^{4}}{4}+\gamma \frac{q^{6}}{6},
\end{aligned}
$$

where $q$ moves on the line, $q \in \mathbb{R}$. Topology of the $q$ space has a sharp contrast with the $\alpha$-HMF model in which $q$ is on the unit circle. From an analogy of the $\alpha$-Hamiltonian mean-field model and the conventional naming of the FPUT models, we call this model the $\alpha$-FPUT- $\beta \gamma$ model. To realize the soft springs, the coefficients are fixed as $\beta=-2$ and $\gamma=1$, which give plateaus around $x= \pm 1$. The fixed boundary condition is given as described in Sec. IV B. Spatially localized periodic solutions of the ST, P-in-phase, and P-antiphase modes are obtained by the shooting method with fixing the period $T=9$ and the parameter $\alpha=0.5$, and they are exhibited in Fig. 5 . We note that the corresponding frequency $\omega \simeq 0.7$ is in the lower gap, as confirmed in Fig. 2(b). The periodic solutions are continued from $\alpha=0.5$ to other values of $\alpha$ by varying $\alpha$ with the step size $\Delta \alpha=10^{-4}$, while we keep the target period as $T=9$. The spatial localization and the stability of the obtained periodic solutions are reported in Fig. 6. Here the spatial localization is revealed by computing the first and second largest amplitudes, and the stability is investigated by the maximum absolute value $\mu_{\max }$ of the characteristic multipliers. Qualitatively, the initial configurations and dynamics of DBs are similar to the $\alpha$-Hamiltonian mean-field model. We remark that the drastic increase of instability appears at a smaller $\alpha$ than the $\alpha$-Hamiltonian mean-field case with the frequency $\omega \simeq 0.42$. This is consistent with the fact that, in the $\alpha$ FPUT- $\beta \gamma$ model with $\omega=0.7$, the lower gap disappears at a smaller $\alpha$.

\section{SUMMARY AND DISCUSSION}

We have proposed a mechanism, the long-range couplings, to open a lower gap without on-site potential. The two theorems under the periodic boundary condition imply that this mechanism is universal for any long-range lattice system, irrespective of details of the coupling functions $\phi(q)$, while the lower gap disappears in short-range systems. In the $\alpha$-HMF model, which is in classical correspondence to the quantum $X Y$ spin systems realized experimentally [28], the existence of spatially localized periodic solutions has been numerically demonstrated with analyses of stability. The numerical results suggest that the 

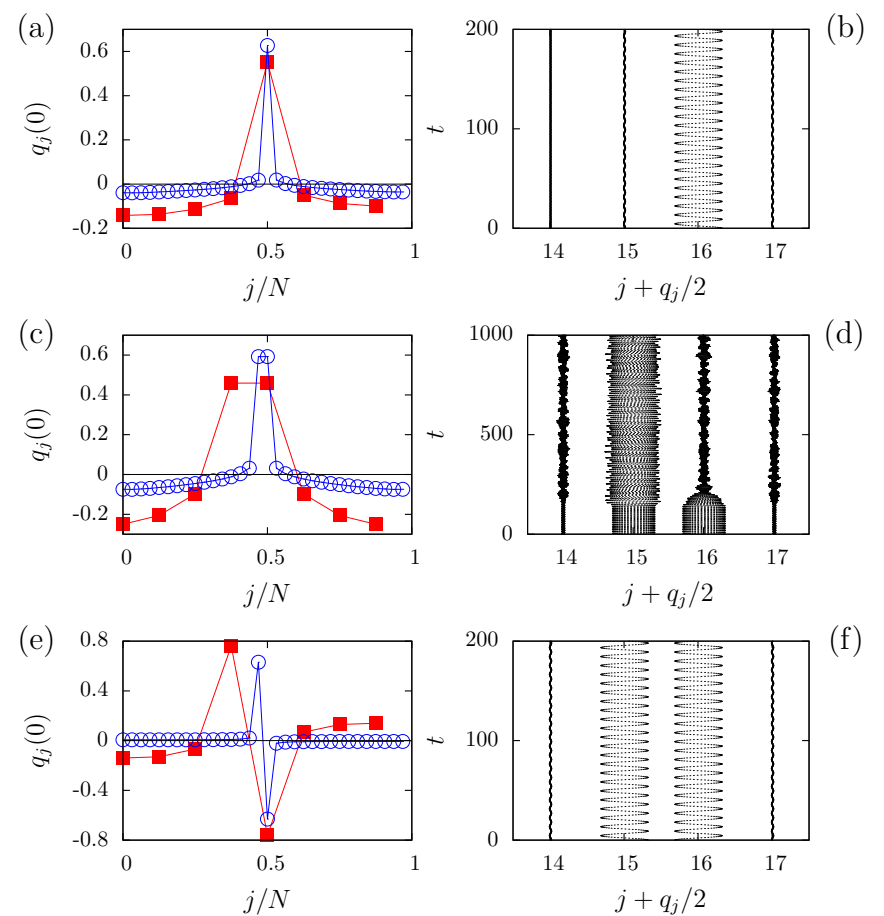

(d)

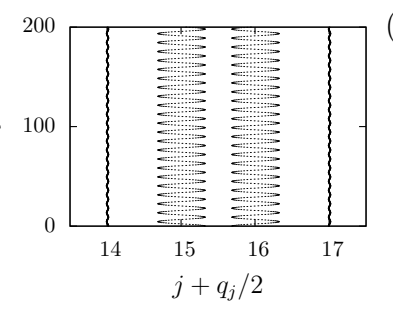

(f)
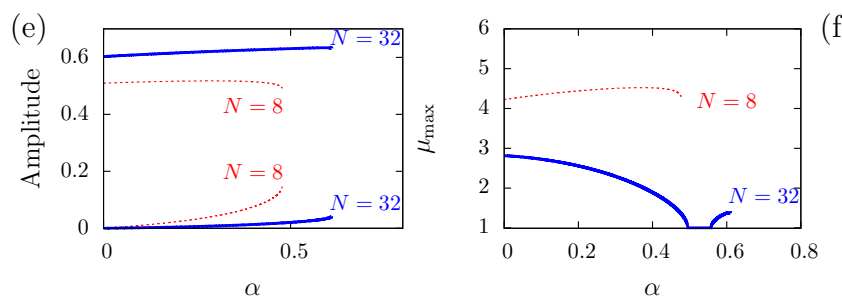

(f)

FIG. 6. The $\alpha$ dependence of the (a) and (b) ST, (c) and (d) $\mathrm{P}$-in-phase, and (e) and (f) P-antiphase modes, which are continued from $\alpha=0.5$ reported in Fig. 5, for $N=8$ (red thin dotted lines) and $N=32$ (blue thick solid lines). The period is $T=9 \mathrm{in}$ all the panels. (a), (c), and (e) The first (upper) and second (lower) largest amplitudes. (b), (d), and (f) Maximum absolute value of the characteristic multipliers.

The spatial localization is enhanced as the number of particles increases. This is consistent with the long-range nature, which is actualized by interacting with many particles. In the limit of large population, the classical dynamics of long-range systems, including the models considered with $\alpha<1$, is described by the Vlasov equation [47]. The Vlasov equation is a partial differential equation for the one-particle distribution function and has an infinite number of Casimir invariants. The Casimir invariants impose constraints on the dynamics and cause strange phenomena: Critical exponents differ from the equilibrium statistical mechanics [48-50] and the finite-size fluctuation evolves from one level to the thermal equilibrium level even though the initial conditions are in thermal equilibrium [51]. We may therefore expect some strange phenomena of DBs in long-range systems due to the Casimir invariants; they are yet to be revealed.

Finally, we stress that introducing long-range couplings is a different idea to excite or maintain DBs with low frequencies. We expect this idea to motivate successive research and create connections among material science, information theory, and nonlinear dynamical theory.

\section{ACKNOWLEDGMENTS}

Y.Y.Y. acknowledges support from JSPS KAKENHI through Grant No. 16K05472. Y.D. acknowledges support from JSPS KAKENHI through Grant No. 16K05041. 


\section{APPENDIX A: PROOFS OF THEOREMS 1 AND 2}

\section{Notation}

Introducing

$$
S(N)=\sum_{k=1}^{N / 2-1} J_{k}, \quad T(N)=\sum_{k=1}^{N / 2-1} J_{k} \sin ^{2} \frac{\pi k}{N},
$$

we can rewrite $\lambda_{1}(N)$ as

$$
\lambda_{1}(N)=\frac{4 T(N)+2 J_{N / 2}}{2 S(N)+J_{0}+J_{N / 2}} .
$$

For the proofs, we further introduce the terms

$$
S_{\alpha}(N)=\sum_{k=1}^{N / 2-1} \frac{1}{k^{\alpha}}, \quad T_{\alpha}(N)=\sum_{k=1}^{N / 2-1} \frac{1}{k^{\alpha}} \sin ^{2} \frac{\pi k}{N}, \quad \alpha \geqslant 0
$$

and the functions

$$
f_{\alpha}(x)=\frac{1}{x^{\alpha}}, \quad g_{\alpha}(x)=\frac{1}{x^{\alpha}} \sin ^{2}(\pi x), \quad \alpha \geqslant 0 .
$$

\section{Lemmas}

For proving the two theorems, we show three lemmas which give estimations of $S_{\alpha}(N)$ and $T_{\alpha}(N)$.

Lemma 1. Let $\alpha \geqslant 0$. Then the following inequalities hold:

$$
\begin{aligned}
& N^{1-\alpha} \int_{1 / N}^{1 / 2} f_{\alpha}(x) d x \leqslant S_{\alpha}(N) \\
& \leqslant 1+N^{1-\alpha} \int_{2 / N}^{1 / 2} f_{\alpha}(x-1 / N) d x .
\end{aligned}
$$

Proof. The summation $S_{\alpha}(N)$ can be written as

$$
S_{\alpha}(N)=N^{1-\alpha} \sum_{k=1}^{N / 2-1} \frac{1}{N} f_{\alpha}\left(\frac{k}{N}\right)
$$

Since the function $f_{\alpha}(x)$ is a positive and monotonically decreasing function, we have

$$
f_{\alpha}(x) \leqslant f_{\alpha}(k / N) \leqslant f_{\alpha}(x-1 / N), \quad x \in[k / N,(k+1) / N] .
$$

Performing the integration in $[k / N,(k+1) / N]$, summing up from $k=2$ to $k=N / 2-1$, and multiplying $N^{1-\alpha}$, we have

$$
\begin{aligned}
N^{1-\alpha} \int_{2 / N}^{1 / 2} f_{\alpha}(x) d x & \leqslant N^{1-\alpha} \sum_{k=2}^{N / 2-1} \frac{1}{N} f_{\alpha}\left(\frac{k}{N}\right) \\
& \leqslant N^{1-\alpha} \int_{2 / N}^{1 / 2} f_{\alpha}(x-1 / N) d x .
\end{aligned}
$$

The contribution from $k=1$ is estimated as

$$
N^{1-\alpha} \int_{1 / N}^{2 / N} f_{\alpha}(x) d x \leqslant N^{1-\alpha} \frac{1}{N} f_{\alpha}\left(\frac{1}{N}\right)=1
$$

and therefore Lemma 1 has been proven.

Lemma 2. Let $I$ be the interval $I=(0,1 / 2)$. Here $g_{\alpha}(x)$ is positive in $I$. For $\alpha \geqslant 2$, the function $g_{\alpha}(x)$ is a monotonically decreasing function in $I$. For $\alpha=0$, the function $g_{\alpha}(x)$ is a monotonically increasing function in $I$. For $0<\alpha<2$, the function $g_{\alpha}(x)$ has the unique critical point $x_{\alpha} \in I$ and monotonically increases (decreases) in $\left(0, x_{\alpha}\right)\left[\left(x_{\alpha}, 1 / 2\right)\right]$.

Proof. The positiveness of $g_{\alpha}(x)$ in $I$ is obvious. The derivative of $g_{\alpha}(x)$ is

$$
\begin{aligned}
& g_{\alpha}^{\prime}(x)=x^{-\alpha-1} \sin (\pi x) \cos (\pi x) h_{\alpha}(x), \\
& h_{\alpha}(x)=2 \pi x-\alpha \tan (\pi x)
\end{aligned}
$$

We have $x^{-\alpha-1} \sin (\pi x) \cos (\pi x)>0$ in $I$ and we focus on the sign of $h_{\alpha}(x)$. We have $h_{\alpha}(x)<0$ for $\alpha \geqslant 2$ and $h_{\alpha}(x)>0$ for $\alpha=0$ in $I$. For $0<\alpha<2, h_{\alpha}(x)$ has the unique zero point $x_{\alpha}$ and $h_{\alpha}(x)>0$ in $\left(0, x_{\alpha}\right)$ and $h_{\alpha}(x)<0$ in $\left(x_{\alpha}, 1 / 2\right)$. Therefore, Lemma 2 has been proven.

We remark that, from the fact $\sin ^{2}(\pi x) \leqslant(\pi x)^{2}$ in $x \in$ $[0,1 / 2]$, we have $0 \leqslant g_{\alpha}(x)=x^{-\alpha} \sin ^{2}(\pi x) \leqslant \pi^{2} x^{2-\alpha}$ and $\lim _{x \rightarrow+0} g_{\alpha}(x)=0$ for $\alpha<2$. This is consistent with Lemma 2 , which implies that $g_{\alpha}(x)$ takes a maximum value at $x_{\alpha}$ for $0<\alpha<2$ and that $g_{\alpha}(x)$ monotonically increases for $\alpha=0$.

Lemma 3. Let $\alpha \geqslant 0$. Let $x_{\alpha}(0<\alpha<2)$ be the unique critical point mentioned in Lemma 2 and let $x_{0}=1 / 2$. For $\alpha \geqslant 2$, the inequalities

$$
N^{1-\alpha} \int_{1 / N}^{1 / 2} g_{\alpha}(x) d x \leqslant T_{\alpha}(N) \leqslant \sin ^{2} \frac{\pi}{N}+N^{1-\alpha} \int_{2 / N}^{1 / 2} g_{\alpha}(x-1 / N) d x
$$

hold. For $0 \leqslant \alpha<2$, the inequalities

$$
N^{1-\alpha}\left[\int_{1 / N}^{x_{\alpha}} g_{\alpha}(x-1 / N) d x+\int_{x_{\alpha}+1 / N}^{1 / 2} g_{\alpha}(x) d x\right] \leqslant T_{\alpha}(N) \leqslant N^{1-\alpha}\left[\frac{1}{N} g_{\alpha}\left(x_{\alpha}\right)+\int_{1 / N}^{1 / 2} g_{\alpha}(x) d x\right]
$$

hold.

Proof. For $\alpha \geqslant 2, g_{\alpha}(x)$ monotonically decreases and the same strategy as for Lemma 1 results in the inequalities (A11). For $0 \leqslant \alpha<2$, we separately discuss the three cases with introducing the notation of the interval $I_{k}=[k / N,(k+1) / N]$.

(i) Case $0<\alpha<2$ and $x_{\alpha}<1 / 2-1 / N$. We have

$$
\begin{gathered}
g_{\alpha}(x-1 / N) \leqslant g_{\alpha}(k / N) \leqslant g_{\alpha}(x), \quad x \in I_{k} \cap\left(0, x_{\alpha}\right), \quad 0 \leqslant g_{\alpha}(k / N) \leqslant g_{\alpha}\left(x_{\alpha}\right), \quad x \in\left(x_{\alpha}, x_{\alpha}+1 / N\right) \\
g_{\alpha}(x) \leqslant g_{\alpha}(k / N) \leqslant g_{\alpha}(x-1 / N), \quad x \in I_{k} \cap\left(x_{\alpha}+1 / N, 1 / 2\right)
\end{gathered}
$$


and hence, from $g_{\alpha}(x) \geqslant 0$, we have

$$
\begin{aligned}
N^{1-\alpha}\left[\int_{1 / N}^{x_{\alpha}} g_{\alpha}(x-1 / N) d x+\int_{x_{\alpha}+1 / N}^{1 / 2} g_{\alpha}(x) d x\right] \leqslant T_{\alpha}(N) & \leqslant N^{1-\alpha}\left[\frac{1}{N} g_{\alpha}\left(x_{\alpha}\right)+\int_{1 / N}^{x_{\alpha}} g_{\alpha}(x) d x+\int_{x_{\alpha}+1 / N}^{1 / 2} g_{\alpha}(x-1 / N) d x\right] \\
& \leqslant N^{1-\alpha}\left[\frac{1}{N} g_{\alpha}\left(x_{\alpha}\right)+\int_{1 / N}^{1 / 2} g_{\alpha}(x) d x\right] .
\end{aligned}
$$

(ii) Case $0<\alpha<2$ and $1 / 2-1 / N \leqslant x_{\alpha}<1 / 2$. The estimations are modified as

$$
g_{\alpha}(x-1 / N) \leqslant g_{\alpha}(k / N) \leqslant g_{\alpha}(x), \quad x \in I_{k} \cap\left(0, x_{\alpha}\right), \quad 0 \leqslant g_{\alpha}(k / N) \leqslant g_{\alpha}\left(x_{\alpha}\right), \quad x \in\left(x_{\alpha}, 1 / 2\right)
$$

and hence, noting $\int_{x_{\alpha}+1 / N}^{1 / 2} g_{\alpha}(x) d x \leqslant 0$, we have

$$
\begin{aligned}
N^{1-\alpha}\left[\int_{1 / N}^{x_{\alpha}} g_{\alpha}(x-1 / N) d x+\int_{x_{\alpha}+1 / N}^{1 / 2} g_{\alpha}(x) d x\right] & \leqslant N^{1-\alpha} \int_{1 / N}^{x_{\alpha}} g_{\alpha}(x-1 / N) d x \leqslant T_{\alpha}(N) \leqslant N^{1-\alpha}\left[\int_{1 / N}^{x_{\alpha}} g_{\alpha}(x) d x+\frac{1 / 2-x_{\alpha}}{N} g_{\alpha}\left(x_{\alpha}\right)\right] \\
& \leqslant N^{1-\alpha}\left[\frac{1}{N} g_{\alpha}\left(x_{\alpha}\right)+\int_{1 / N}^{1 / 2} g_{\alpha}(x) d x\right] .
\end{aligned}
$$

(iii) Case $\alpha=0$. The function $g_{\alpha}(x)$ monotonically increases and the estimations become

$$
g_{\alpha}(x-1 / N) \leqslant g_{\alpha}(k / N) \leqslant g_{\alpha}(x), \quad x \in I_{k} .
$$

Thus, we have

$$
\begin{aligned}
N^{1-\alpha}\left[\int_{1 / N}^{x_{\alpha}} g_{\alpha}(x-1 / N) d x+\int_{x_{\alpha}+1 / N}^{1 / 2} g_{\alpha}(x) d x\right] & \leqslant N^{1-\alpha} \int_{1 / N}^{1 / 2} g_{\alpha}(x-1 / N) d x \leqslant T_{\alpha}(N) \\
& \leqslant N^{1-\alpha} \int_{1 / N}^{1 / 2} g_{\alpha}(x) d x \leqslant N^{1-\alpha}\left[\frac{1}{N} g_{\alpha}\left(x_{\alpha}\right)+\int_{1 / N}^{1 / 2} g_{\alpha}(x) d x\right] .
\end{aligned}
$$

All three cases satisfy (A12) and Lemma 3 has been proven.

\section{Proof of Theorem 1}

From Lemma 1 and $\alpha>1, S(N)$ is estimated as

$$
0<S(N) \leqslant J S_{\alpha}(N) \leqslant J\left[1+N^{1-\alpha} \frac{(1 / 2-1 / N)^{1-\alpha}-(1 / N)^{1-\alpha}}{1-\alpha}\right]=J \frac{(N / 2)^{1-\alpha}-\alpha}{1-\alpha} .
$$

From the assumption (13), $S(N)$ is positive in the limit $N \rightarrow \infty$ and is bounded as

$$
0<\lim _{N \rightarrow \infty} S(N) \leqslant J \frac{\alpha}{\alpha-1} .
$$

Since the denominator of (A2) is bounded and $J_{N / 2}$, which is smaller than $J /(N / 2)^{\alpha}$, goes to 0 in the limit $N \rightarrow \infty$, it is enough to show $\lim _{N \rightarrow \infty} T(N)=0$.

From the assumption $J_{k} \leqslant J / k^{\alpha}, T(N)$ is estimated as

$$
0 \leqslant T(N) \leqslant J T_{\alpha}(N) \text {. }
$$

We separately discuss the two cases (i) $\alpha \geqslant 2$ and (ii) $1<\alpha<2$.

(i) Case $\alpha \geqslant 2$. Lemma 3 gives

$$
\begin{aligned}
0 & \leqslant T(N) \leqslant J\left[\sin ^{2} \frac{\pi}{N}+N^{1-\alpha} \int_{1 / N}^{1 / 2-1 / N} x^{-\alpha} \sin ^{2}(\pi x) d x\right] \leqslant J\left[\sin ^{2} \frac{\pi}{N}+N^{1-\alpha} \pi^{2} \int_{1 / N}^{1 / 2-1 / N} x^{2-\alpha}\right] \\
& = \begin{cases}J\left[\sin ^{2} \frac{\pi}{N}+N^{1-\alpha} \pi^{2} \frac{(1 / 2-1 / N)^{3-\alpha}-(1 / N)^{3-\alpha}}{3-\alpha}\right] & (\alpha \neq 3) \\
J\left[\sin ^{2} \frac{\pi}{N}+N^{1-\alpha} \pi^{2} \ln (N / 2-1)\right] & (\alpha=3) .\end{cases}
\end{aligned}
$$

In this way we used the fact $\sin ^{2}(\pi x) \leqslant(\pi x)^{2}$ in $x \in(0,1 / 2)$. Further, using the fact that $\lim _{N \rightarrow \infty} N^{-2} \ln N=0$, we have $\lim _{N \rightarrow \infty} T(N)=0$.

(ii) Case $1<\alpha<2$. Lemma 3 gives

$$
0 \leqslant T(N) \leqslant N^{1-\alpha}\left[\frac{1}{N} g_{\alpha}\left(x_{\alpha}\right)+\int_{1 / N}^{1 / 2} g_{\alpha}(x) d x\right] \leqslant N^{1-\alpha}\left[\frac{1}{N} g_{\alpha}\left(x_{\alpha}\right)+\pi^{2} \frac{(1 / 2)^{3-\alpha}-(1 / N)^{3-\alpha}}{3-\alpha}\right] .
$$

Therefore, we have $\lim _{N \rightarrow \infty} T(N)=0$. 


\section{Proof of Theorem 2}

From the assumption $J^{\prime} / K^{\alpha} \leqslant J_{k} \leqslant J / k^{\alpha}$, we have

$$
J^{\prime} S_{\alpha}(N) \leqslant S(N) \leqslant J S_{\alpha}(N), \quad J^{\prime} T_{\alpha}(N) \leqslant T(N) \leqslant J T_{\alpha}(N) .
$$

The first eigenvalue $\lambda_{1}(N)$ is therefore bounded as

$$
\frac{4 J^{\prime} T_{\alpha}(N)+2 J_{N / 2}}{2 J S_{\alpha}(N)+J_{0}+J_{N / 2}} \leqslant \lambda_{1}(N) \leqslant \frac{4 J T_{\alpha}(N)+2 J_{N / 2}}{2 J^{\prime} S_{\alpha}(N)+J_{0}+J_{N / 2}} .
$$

The above estimations are modified by Lemmas 1 and 3 as

$$
\frac{4 J^{\prime} N^{1-\alpha}\left[\int_{1 / N}^{x_{\alpha}} g_{\alpha}(x-1 / N) d x+\int_{x_{\alpha}+1 / N}^{1 / 2} g_{\alpha}(x) d x\right]+2 J_{N / 2}}{2 J\left[1+N^{1-\alpha} \int_{2 / N}^{1 / 2} f_{\alpha}(x-1 / N) d x\right]+J_{0}+J_{N / 2}} \leqslant \lambda_{1}(N) \leqslant \frac{4 J N^{1-\alpha}\left[\frac{1}{N} g_{\alpha}\left(x_{\alpha}\right)+\int_{1 / N}^{1 / 2} g_{\alpha}(x) d x\right]+2 J_{N / 2}}{2 J^{\prime} N^{1-\alpha} \int_{1 / N}^{1 / 2} f_{\alpha}(x) d x+J_{0}+J_{N / 2}} .
$$

We separately discuss the two cases (i) $0 \leqslant \alpha<1$ and (ii) $\alpha=1$.

Case (i) $0 \leqslant \alpha<1$. Taking the limit $N \rightarrow \infty$ in (A26), we have

$$
\frac{4 J^{\prime} \int_{0}^{1 / 2} g_{\alpha}(x) d x}{2 J \int_{0}^{1 / 2} f_{\alpha}(x) d x} \leqslant \lim _{N \rightarrow \infty} \lambda_{1}(N) \leqslant \frac{4 J \int_{0}^{1 / 2} g_{\alpha}(x) d x}{2 J^{\prime} \int_{0}^{1 / 2} f_{\alpha}(x) d x} .
$$

Performing the integral appearing in the denominators, the estimations (15) can be shown.

Case (ii) $\alpha=1$. In the limit $N \rightarrow \infty$, the integral $\int_{1 / N}^{1 / 2} g_{\alpha}(x) d x$ is bounded as

$$
0 \leqslant \int_{1 / N}^{1 / 2} g_{\alpha}(x) d x \leqslant \pi^{2} \int_{1 / N}^{1 / 2} x d x=\frac{\pi^{2}}{2}\left(\frac{1}{2^{2}}-\frac{1}{N^{2}}\right) .
$$

In contrast, the integral $\int_{1 / N}^{1 / 2} f_{\alpha}(x) d x$ diverges as

$$
\int_{1 / N}^{1 / 2} f_{\alpha}(x) d x=\ln \frac{1}{2}-\frac{1}{N}=\ln \frac{N}{2} .
$$

Therefore, we have $\lim _{N \rightarrow \infty} \lambda_{1}(N)=0$. This asymptotic value satisfies the inequalities (15) with $\alpha=1$.

\section{APPENDIX B: SHOOTING METHOD}

Let us search for a periodic solution with the period $T$ in the dynamical system

$$
\frac{d x}{d t}=f(x, t), \quad x, f \in \mathbb{R}^{n}
$$

by the shooting method. We denote the initial point at $t=0$ by $x_{0}$ and the corresponding orbit in (B1) is defined as

$$
\phi\left(t ; x_{0}\right)=x_{0}+\int_{0}^{t} f\left(\phi\left(s ; x_{0}\right), s\right) d s .
$$

The basic idea is to use the Newton-Raphson method by introducing the evaluation function

$$
F\left(x_{0}, T\right)=\phi\left(t ; x_{0}\right)-x_{0}=\int_{0}^{T} f\left(\phi\left(t ; x_{0}\right), t\right) d t .
$$

This function should be zero if $\phi\left(t ; x_{0}\right)$ represents a periodic solution with the period $T$.

To perform the Newton-Raphson method, we first set an initial trial $x_{0}$. The increment $\delta x$ for the next trial $x_{0}^{\prime}=x_{0}+\delta x$ is computed by solving the equation

$$
D_{x_{0}} F\left(x_{0}, T\right) \delta x=-F\left(x_{0}, T\right),
$$

where $D_{x_{0}} F$ is the Jacobi matrix with respect to $x_{0}$. We repeat this procedure. If the trial points converge to a certain point, then the converged point gives an initial point of a periodic solution.

To realize the above procedure, we need $F\left(x_{0}, T\right)$ and $D_{x_{0}} F\left(x_{0}, T\right)$. Here $F\left(x_{0}, T\right)$ can be obtained by numerically performing the integration of (B3). The Jacobi matrix is expressed as

$$
D_{x_{0}} F\left(x_{0}, T\right)=D_{x_{0}} \phi\left(T ; x_{0}\right)-E_{n} .
$$


We show that the right-hand side of (B5) can be obtained by numerically integrating the linearized equation along the orbit $\phi\left(t ; x_{0}\right)$, which is written as

$$
\frac{d \xi}{d t}=D_{x} f\left(\phi\left(t ; x_{0}\right), t\right) \xi
$$

The solution $\xi\left(t ; x_{0}\right)$ to (B6) with the initial condition $\xi\left(0 ; x_{0}\right)=\xi_{0}$ is

$$
\xi\left(t ; x_{0}\right)=\xi_{0}+\int_{0}^{t} D_{x} f\left(\phi\left(s ; x_{0}\right), t\right) \xi\left(s ; x_{0}\right) d s .
$$

We have $n$ independent solutions to the linearized equation (B6) and they can be arranged in matrix form as

$$
\left(\xi^{(1)}\left(T ; x_{0}\right), \quad \ldots, \quad \xi^{(n)}\left(T ; x_{0}\right)\right)=\left(\xi_{0}^{(1)}, \quad \ldots, \quad \xi_{0}^{(n)}\right)+\int_{0}^{T} D_{x} f\left(\phi\left(t ; x_{0}\right), t\right)\left(\xi^{(1)}\left(t ; x_{0}\right), \quad \ldots, \quad \xi^{(n)}\left(t ; x_{0}\right)\right) d t .
$$

On the other hand, deriving (B2) with respect to $x_{0}$, we have

$$
D_{x_{0}} \phi\left(T ; x_{0}\right)=E_{n}+\int_{0}^{T} D_{x} f\left(\phi\left(t ; x_{0}\right), t\right) D_{x_{0}} \phi\left(t ; x_{0}\right) d t .
$$

Comparing (B8) and (B9), we find that $D_{x_{0}} \phi\left(T ; x_{0}\right)$ is obtained by setting the initial conditions as

$$
\left(\xi_{0}^{(1)}, \quad \ldots, \quad \xi_{0}^{(n)}\right)=E_{n} .
$$

Fortunately, the solution matrix $\left(\xi^{(1)}\left(T ; x_{0}\right), \ldots, \xi^{(n)}\left(T ; x_{0}\right)\right)$ to the linearized equation (B6) simultaneously gives the characteristic multipliers as the eigenvalues.

[1] A. J. Sievers and S. Takeno, Intrinsic Localized Modes in Anharmonic Crystals, Phys. Rev. Lett. 61, 970 (1988).

[2] J. B. Page, Asymptotic solutions for localized vibrational modes in strongly anharmonic periodic systems, Phys. Rev. B 41, 7835 (1990).

[3] P. G. Kevrekidis, K. O. Rasmussen, and A. R. Bishop, Twodimensional discrete breathers: Construction, stability, and bifurcations, Phys. Rev. E 61, 2006 (2000).

[4] D. K. Campbell, S. Flach, and Y. S. Kivshar, Localizing energy through nonlinearity and discreteness, Phys. Today 57 (1), 43 (2004).

[5] S. Aubry, Discrete breathers: Localization and transfer of energy in discrete Hamiltonian nonlinear systems, Physica D 216, 1 (2006).

[6] S. Flach and A. V. Gorbach, Discrete breathers-Advances in theory and applications, Phys. Rep. 467, 1 (2008).

[7] K. Yoshimura, Y. Doi, and M. Kimura, in Progress in Nanophotonics 3, edited by M. Ohtsu and T. Yatsui (Springer International, Cham, 2015), pp. 119-166.

[8] L. Q. English, F. Palmero, P. Candiani, J. Cuevas, R. CarreteroGonzález, P. G. Kevrekidis, and A. J. Sievers, Generation of Localized Modes in an Electrical Lattice Using Subharmonic Driving, Phys. Rev. Lett. 108, 084101 (2012).

[9] L. Q. English, F. Palmero, J. F. Stormes, J. Cuevas, R. CarreteroGonzález, and P. G. Kevrekidis, Nonlinear localized modes in two-dimensional electrical lattices, Phys. Rev. E 88, 022912 (2013).

[10] M. Sato, B. E. Hubbard, A. J. Sievers, B. Ilic, D. A. Czaplewski, and H. G. Craighead, Observation of Locked Intrinsic Localized Vibrational Modes in a Micromechanical Oscillator Array, Phys. Rev. Lett. 90, 044102 (2003).

[11] M. Kimura and T. Hikihara, Capture and release of traveling intrinsic localized mode in coupled cantilever array, Chaos 109, 013138 (2009).
[12] N. Boechler, G. Theocharis, S. Job, P. G. Kevrekidis, M. A. Porter, and C. Daraio, Discrete Breathers in One-Dimensional Diatomic Granular Crystals, Phys. Rev. Lett. 104, 244302 (2010).

[13] G. James, P. G. Kevrekidis, and J. Cuevas, Breathers in oscillator chains with Hertzian interactions, Physica D 251, 39 (2013).

[14] S. V. Dmitriev, A. P. Chetverikov, and M. G. Velarde, Discrete breathers in 2D and 3D crystals, Phys. Status Solidi B 252, 1682 (2015).

[15] J. Bajars, J. C. Eilbeck, and B. Leimkuhler, in Quodons in Mica, edited by J. F. R. Archilla, N. Jiménez, V. J. Sánchez-Morcillo, and L. M. García-Raffi (Springer International, Cham, 2015), pp. 35-67.

[16] R. Lai and A. J. Sievers, Intrinsic localized spin wave modes in easy-axis antiferromagnetic chains, J. Appl. Phys. 81, 3972 (1997).

[17] U. T. Schwarz, L. Q. English, and A. J. Sievers, Experimental Generation and Observation of Intrinsic Localized Spin Wave Modes in an Antiferromagnet, Phys. Rev. Lett. 83, 223 (1999).

[18] Y. Zolotaryuk, S. Flach, and V. Fleurov, Discrete breathers in classical spin lattices, Phys. Rev. B 63, 214422 (2001).

[19] B. Tang, D.-J. Li, and Y. Tang, Spin discrete breathers in two-dimensional square anisotropic ferromagnets, Phys. Scr. 89, 095208 (2014).

[20] M. Lakshmanan, B. Subash, and A. Saxena, Intrinsic localized modes of a classical discrete anisotropic Heisenberg ferromagnetic spin chain, Phys. Lett. A 378, 1119 (2014).

[21] Y. Kawai, Y. Kiwamoto, K. Ito, A. Sanpei, Y. Soga, J. Aoki, and K. Itoh, Relaxation of azimuthal flow pattern from ring to bell shape through two-dimensional turbulence triggered by diocotron instability, J. Phys. Soc. Jpn. 75, 104502 (2006).

[22] Y. Yatsuyanagi, Y. Kiwamoto, T. Ebisuzaki, T. Hatori, and T. Kato, Simulations of diocotron instability using a specialpurpose computer, MDGRAPE-2, Phys. Plasmas 10, 3188 (2003). 
[23] T. Watanabe, T. Iwayama, and H. Fujisaka, Scaling law for coherent vortices in decaying drift Rossby wave turbulence, Phys. Rev. E 57, 1636 (1998).

[24] D. Porras and J. I. Cirac, Effective Quantum Spin Systems with Trapped Ions, Phys. Rev. Lett. 92, 207901 (2004).

[25] K. Kim, M.-S. Chang, R. Islam, S. Korenblit, L.-M. Duan, and C. Monroe, Entanglement and Tunable Spin-Spin Couplings between Trapped Ions Using Multiple Transverse Modes, Phys. Rev. Lett. 103, 120502 (2009).

[26] J. W. Britton, B. C. Sawyer, A. C. Keith, C.-C. J. Wang, J. K. Freericks, H. Uys, M. J. Biercuk, J. J. John, and J. Bollinger, Engineered two-dimensional Ising interactions in a trapped-ion quantum simulator with hundreds of spins, Nature (London) 484 , 489 (2012).

[27] R. Islam, C. Senko, W. C. Campbell, S. Korenblit, J. Smith, A. Lee, E. E. Edwards, C.-C. J. Wang, J. K. Freericks, and C. Monroe, Emergence and frustration of magnetism with variablerange interactions in a quantum simulator, Science 340, 583 (2013).

[28] P. Richerme, Z.-X. Gong, A. Lee, C. Senko, J. Smith, M. FossFeig, S. Michalakis, A. V. Gorshkov, and C. Monroe, Non-local propagation of correlations in quantum systems with long-range interactions, Nature (London) 511, 198 (2014).

[29] P. Hauke and L. Tagliacozzo, Spread of Correlations in LongRange Interacting Quantum Systems, Phys. Rev. Lett. 111, 207202 (2013).

[30] J. Eisert, M. van den Worm, S. R. Manmana, and M. Kastner, Breakdown of Quasilocality in Long-Range Quantum Lattice Models, Phys. Rev. Lett. 111, 260401 (2013).

[31] E. Lieb and D. Robinson, The finite group velocity of quantum spin systems, Commun. Math. Phys. 28, 251 (1972).

[32] Z.-X. Gong, M. Foss-Feig, S. Michalakis, and A. V. Gorshkov, Persistence of Locality in Systems with Power-Law Interactions, Phys. Rev. Lett. 113, 030602 (2014).

[33] K. Hori and S. Takeno, Moving self-localized modes for the displacement field in a one-dimensional lattice system with quartic anharmonicity, J. Phys. Soc. Jpn. 51, 2186 (1992).

[34] D. Chen, S. Aubry, and G. P. Tsironis, Breather Mobility in Discrete $\phi^{4}$ Nonlinear Lattices, Phys. Rev. Lett. 77, 4776 (1996).

[35] S. Flach, Breathers on lattices with long range interaction, Phys. Rev. E 58, R4116 (1998).

[36] G. Miloshevich, J. P. Nguenang, T. Dauxois, R. Khomeriki, and S. Ruffo, Traveling solitons in long-range oscillator chains, J. Phys. A: Math. Theor. 50, 12LT02 (2017).
[37] J. Cuevas, J. F. R. Archilla, Y. B. Gaididei, and F. R. Romero, Moving breathers in a DNA model with competing shortand long-range dispersive interactions, Physica D 163, 106 (2002).

[38] R. S. MacKay and S. Aubry, Proof of existence of breathers for time-reversible or Hamiltonian networks of weakly coupled oscillators, Nonlinearity 7, 1623 (1994).

[39] J. L. Marín and S. Aubry, Breathers in nonlinear lattices: Numerical calculation from the anticontinuous limit, Nonlinearity 9, 1501 (1996).

[40] S. Aubry, Breathers in nonlinear lattices: Existence, linear stability and quantization, Physica D 103, 201 (1997).

[41] C. Anteneodo and C. Tsallis, Breakdown of Exponential Sensitivity to Initial Conditions: Role of the Range of Interactions, Phys. Rev. Lett. 80, 5313 (1998).

[42] E. Fermi, J. Pasta, S. Ulam, Studies of nonlinear problems, Los Alamos Scientific Laboratory report LA-1940 (1955).

[43] T. Dauxois, Fermi, Pasta, Ulam, and a mysterious lady, Phys. Today 61 (1), 55 (2008).

[44] A. Campa, T. Dauxois, and S. Ruffo, Statistical mechanics and dynamics of solvable models with long-range interactions, Phys. Rep. 480, 57 (2009).

[45] A. Campa, A. Giansanti, and D. Moroni, Canonical solution of a system of long-range interacting rotators on a lattice, Phys. Rev. E 62, 303 (2000).

[46] T. Mori, Analysis of the exactness of mean-field theory in long-range interacting systems, Phys. Rev. E 82, 060103(R) (2010).

[47] R. Bachelard, T. Dauxois, G. De Ninno, S. Ruffo, and F. Staniscia, Vlasov equation for long-range interactions on a lattice, Phys. Rev. E 83, 061132 (2011).

[48] S. Ogawa, A. Patelli, and Y. Y. Yamaguchi, Non-mean-field critical exponent in a mean-field model: Dynamics versus statistical mechanics, Phys. Rev. E 89, 032131 (2014).

[49] S. Ogawa and Y. Y. Yamaguchi, Nonlinear response for external field and perturbation in the Vlasov system, Phys. Rev. E 89, 052114 (2014).

[50] S. Ogawa and Y. Y. Yamaguchi, Landau-like theory for universality of critical exponents in quasistationary states of isolated mean-field systems, Phys. Rev. E 91, 062108 (2015).

[51] Y. Y. Yamaguchi, Strange scaling and relaxation of finite-size fluctuation in thermal equilibrium, Phys. Rev. E 94, 012133 (2016). 